\title{
Intracranial hemorrhage complicating anticoagulant prophylactic therapy in three hospitalized COVID-19 patients
}

\author{
Muhammad Usman Ghani ${ }^{1}$ (D) $\cdot$ Mukesh Kumar $^{1} \cdot$ Usman Ghani $^{1} \cdot$ Fnu Sonia ${ }^{1} \cdot$ Syed Ali Abbas ${ }^{1}$
}

Received: 20 May 2020 / Revised: 4 June 2020 / Accepted: 10 June 2020 / Published online: 22 June 2020

(C) Journal of NeuroVirology, Inc. 2020

\begin{abstract}
SARS-CoV2 has led to a global pandemic affecting almost 3 million people in almost over 3 months. Various clinical presentations have been reported so far and no definite therapy is established. Anticoagulation is recommended by several experts to address the potential prothrombotic complications from COVID-19, but its safety and regimen need further clinical trials and safety and efficacy profile. Here, we present three cases of intracranial hemorrhage in three critically ill patients with COVID-19 and discuss their course in relation to various regimens of anticoagulation used.
\end{abstract}

Keywords Intracranial hemorrhage · COVID-19 · Anticoagulation

\section{Introduction}

SARS-CoV2, like other respiratory viruses, may invade the central nervous system (Desforges et al. 2020). As data on COVID-19 is emerging, various neurological manifestations have been observed (Mao et al. 2020). To our knowledge, one case of intraparenchymal hemorrhage has been reported in a patient with COVID-19 in the USA (Vu et al. 2020). Given the reports of thrombotic complications in this disease, anticoagulation protocols have been implemented by many institutions. Here, we present three cases of fatal intracranial hemorrhage in patients with severe SARS-CoV2 infection on therapeutic anticoagulation.

\section{Case presentations}

Patient 1 A 59-year-old man with well-controlled hypertension presented with acute hypoxic respiratory failure from COVID-19 pneumonia and required intubation. The patient was found to have an elevated d-dimer to $>3$ and received

Muhammad Usman Ghani usmanghani162@gmail.com

1 Department of Medicine, Montefiore Medical Center, Albert Einstein College of Medicine, 600 East 233 Street, Bronx, NY 10470, USA therapeutic anticoagulation with unfractionated heparin (UFH). His aPTT remained therapeutic (45-60 s) and systolic blood pressure ranged from 120 to $130 \mathrm{mmHg}$ without medications. On day 18 , he was found to have absent brainstem reflexes. A computed tomography (CT) scan of the head revealed acute subarachnoid and intraparenchymal hemorrhages within the posterior fossa. He had severe cerebral edema and transtentorial, uncal, and tonsillar herniation (Fig. 1). aPTT on the day of CT scan was $57 \mathrm{~s}$. A subsequent apnea test revealed no respiratory effort after $12 \mathrm{~min}$ which is consistent with brain death.

Patient 2 A 61-year-old woman with type 2 diabetes mellitus presented with COVID-19 pneumonia and was intubated. She was found to have acute respiratory distress syndrome (ARDS). Initially, she received prophylactic anticoagulation with apixaban, but was then switched to therapeutic UFH on day 10, as her d-dimer levels were up-trending. Physical examination on day 10 of hospitalization revealed fixed dilated pupils with no brainstem reflexes. CT head showed diffuse brain anoxia with scattered subarachnoid hemorrhages, a subdural hematoma with left to right midline shift and tonsillar herniation (Fig. 2). aPTT at the time of detection of intracranial hemorrhage was $63 \mathrm{~s}$. She was subsequently declared brain-dead.

Patient 3 A 59-year-old woman with hypertension was admitted with COVID-19 pneumonia and intubated for hypoxic respiratory failure. Prophylactic anticoagulation with 


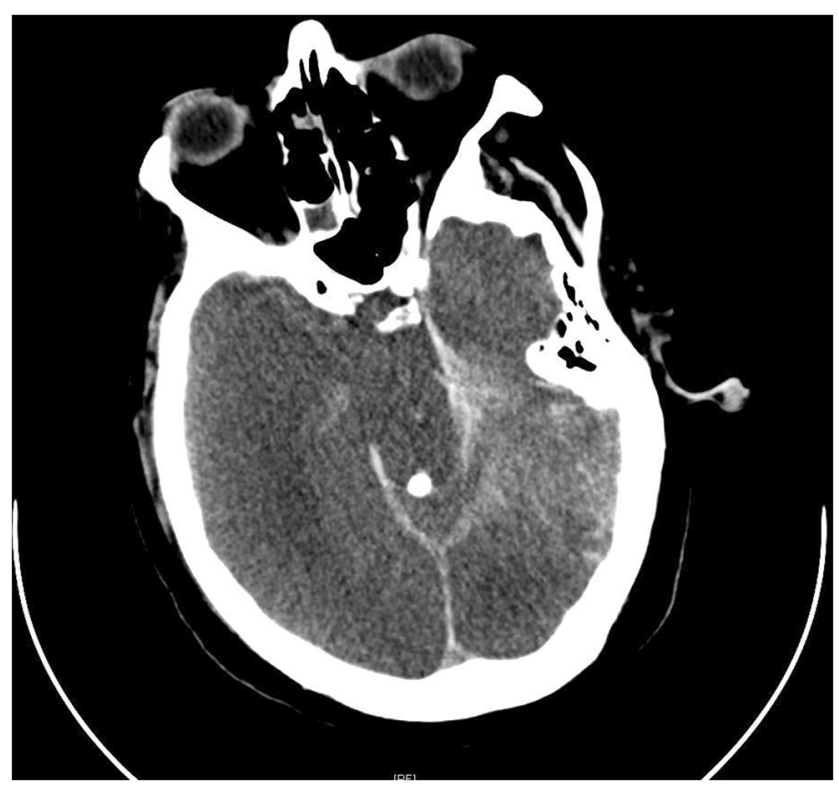

Fig. 1 Acute hypoxic brain injury with severe global cerebral swelling resulting in transtentorial, uncal, and tonsillar herniation. Acute subarachnoid and intraparenchymal hemorrhage within the posterior fossa involving the brainstem

apixaban was started and then switched on day 6 to therapeutic enoxaparin due to rising d-dimer levels. She remained comatose despite being off intravenous sedation for 1 week. CT scan of the head on day 15 revealed a large intraparenchymal hemorrhage and cerebral edema, with transtentorial-herniation causing brainstem compression (Fig. 3). Neurological exam and apnea test were consistent with brain death. Throughout the hospital stay, systolic blood pressure ranged between 125 and $140 \mathrm{mmHg}$.

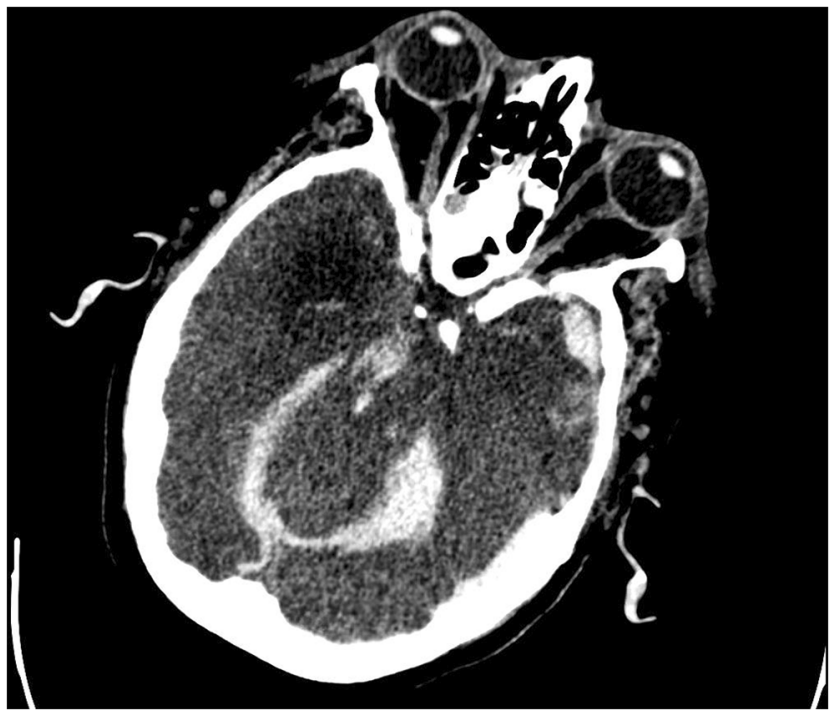

Fig. 2 Diffuse bilateral loss of gray-white matter differentiation consistent with brain anoxia. Diffuse bilateral effacement of the cerebral sulci and cerebellar folia secondary to cerebral edema and scattered bilateral acute subarachnoid hemorrhage

\section{Discussion}

Here, we report three patients with severe COVID-19 who developed intracranial hemorrhage visualized on $\mathrm{CT}$ imaging while on therapeutic anticoagulation. They all had normal baseline neurological status on admission and had no known predisposition to life-threatening bleeding. During their admission, these patients all received steroids, hydroxychloroquine, and anticoagulation per our institutional guidelines at the time of the COVID-19 pandemic.

All patients were found to have cerebral anoxia with variable degrees of intracranial hemorrhage. While patient 3 had the largest area of intracranial hemorrhage, patient 2 suffered from subdural and subarachnoid hemorrhage with midline shift. Cerebral edema and tentorial and transtentorial herniation were common findings in these three patients.

Previous reports during the MERS-CoV epidemic documented similar cases of intracranial hemorrhage (Al-Hameed 2017; Algahtani et al. 2016). In our patients with SARS$\mathrm{CoV} 2$, it is difficult to ascertain whether these neurologic complications were the result of their severe COVID-19 or due to the therapeutic anticoagulation they received. Emerging evidence suggests that coagulopathy is associated with COVID-19 and elevated d-dimer and fibrinogen levels are indicative of a prothrombotic state. The current hematology expert opinion approach toward thromboprophylaxis in hospitalized COVID-19 patients is to avoid therapeutic dosing of anticoagulant therapy unless there is a documented

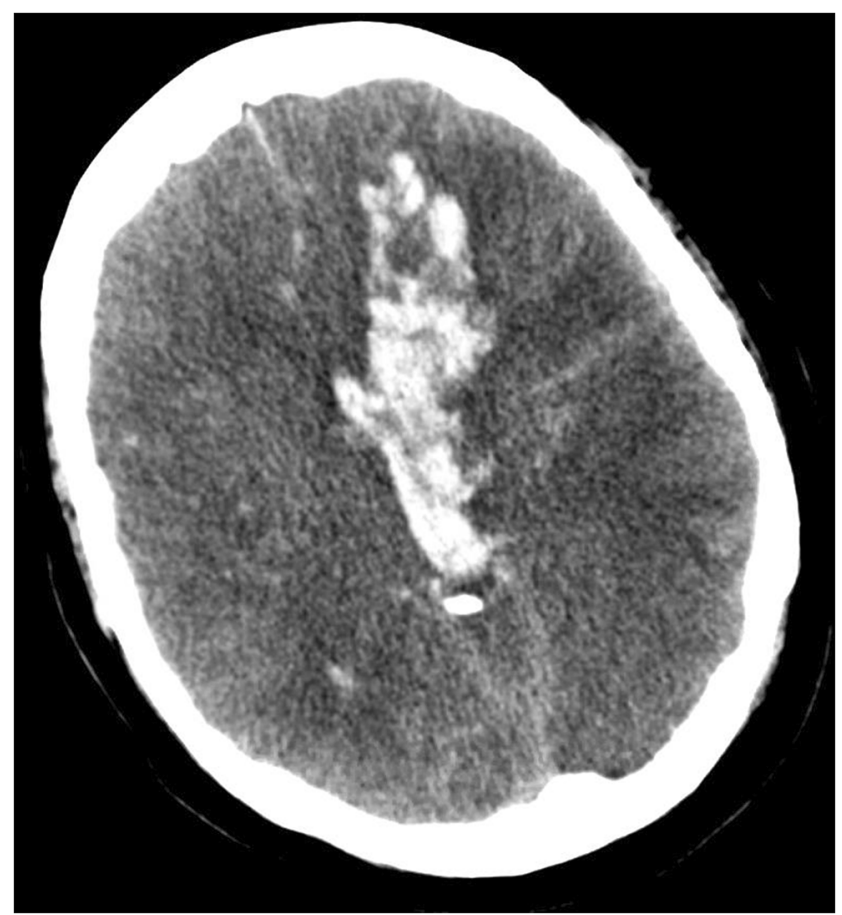

Fig. 3 A large left-sided intraparenchymal hemorrhage with intraventricular extension, superficial subarachnoid blood, and left-sided cerebral swelling 
thrombotic event (Thachil et al. 2020). There is some data suggesting a mortality benefit with the use of anticoagulation in COVID-19 patients (Tang et al. 2020). A prospective multicenter study reported at least $40 \%$ thrombotic complications in patients with COVID-19 (Helms et al. 2020). Further, a recent study reporting neurological manifestations in COVID-19 patients observed cerebrovascular complications including ischemic and hemorrhagic strokes (Mao et al. 2020). At this time, it is unclear which patients will have greater benefit than harm from therapeutic anticoagulation.

All of our patients had intracranial bleeding and it is uncertain if this is a complication from COVID-19 or anticoagulation. Clinicians should remain cautious while starting therapeutic anticoagulation in patients with severe COVID-19 and impaired consciousness. Routine neurological examination and maintaining a low threshold for brain imaging in critically ill patients are imperative to detect lifethreatening intracranial hemorrhagic complications in these patients. More studies are needed to fully elucidate the neurologic complications of COVID-19 and the benefits and risks of therapeutic anticoagulation in patients with COVID-19.

\section{Compliance with ethical standards}

Conflict of interest The authors declare that they have no conflict of interest.

\section{References}

Algahtani H, Subahi A, Shirah B (2016) Neurological complications of Middle East respiratory syndrome coronavirus: a report of two cases and review of the literature. Case Rep Neurol Med 2016:1-6

Al-Hameed FM (2017) Spontaneous intracranial hemorrhage in a patient with Middle East respiratory syndrome coronavirus. Saudi Medi J 38(2):196-200

Desforges M, Le Coupanec A, Dubeau P, Bourgouin A, Lajoie L, Dubé M, Talbot PJ (2020) Human coronaviruses and other respiratory viruses: underestimated opportunistic pathogens of the central nervous system? Viruses 12(1):14

Helms J, Tacquard C, Severac F, Leonard-Lorant I, Ohana M, Delabranche X, Merdji H, Clere-Jehl R, Schenck M, Fagot Gandet F, Fafi-Kremer S (2020) High risk of thrombosis in patients with severe SARS-CoV-2 infection: a multicenter prospective cohort study. Intensive Care Med 4:1-0

Mao L, Jin H, Wang M, Hu Y, Chen S, He Q, Chang J, Hong C, Zhou Y, Wang D, Miao X (2020) Neurologic manifestations of hospitalized patients with coronavirus disease 2019 in Wuhan, China. JAMA Neurol 1;77(6):683-90

Tang N, Bai H, Chen X, Gong J, Li D, Sun Z (2020) Anticoagulant treatment is associated with decreased mortality in severe coronavirus disease 2019 patients with coagulopathy. J Thromb Haemost 18(5):1094-1099

Thachil J, Tang N, Gando S, Falanga A, Cattaneo M, Levi M, Clark C, Iba T (2020) ISTH interim guidance on recognition and management of coagulopathy in COVID-19. J Thromb Haemost 18(5): $1023-1026$

Vu D, Ruggiero M, Choi WS, Masri D, Flyer M, Shyknevsky I, Stein EG (2020) Three unsuspected CT diagnoses of COVID-19. Emerg Radiol 13:1-4

Publisher's note Springer Nature remains neutral with regard to jurisdictional claims in published maps and institutional affiliations. 